\title{
ACF: An Autonomic Communication Framework for Wireless Sensor Networks
}

Jingbo Sun and Rachel Cardell-Oliver

\begin{abstract}
The performance of wireless sensor networks in the field has been shown to be both unreliable and unpredictable. One reason for this is that radio links between pairs of nodes are often unstable, with significant variation in performance over both time and space. Timeout-based, streaming and ExOR protocols, which represent three mechanisms used in improving the reliability in data delivery, are analysed and areas of good and poor performance are identified for certain combinations of packet delivery probabilities on the communication links. A new autonomic communication framework (ACF) is proposed for sensor networks, which is able to adapt to changing environments. Experimental results show that our framework optimises performance under different environmental conditions for performance measures including link efficiency and energy consumption.
\end{abstract}

\section{Introduction}

The performance of sensor network protocols in the field has been shown to be both unreliable and unpredictable $[4,20,19,9]$, leading to low delivery rates and high delivery costs [10]. The main reason for this problem is that sensor nodes use lowpower short-range radio links to communicate with each other. The characteristics of this kind of radio links are significant variation and asymmetry over both time and space $[24,6,19]$, which can cause long periods of poor to no connectivity.

Autonomic systems are those with the ability to self-configure to suit varying and unpredictable conditions, that can monitor themselves constantly for optimal functioning, that are able to find alternate ways to function when they encounter problems, and that are able to adapt to varying environments. The goal of our work

Jingbo Sun and Rachel Cardell-Oliver

School of Computer Science \& Software Engineering and Cooperative Research Centre for Plantbased Management of Dryland Salinity, The University of Western Australia, M002, 35 Stirling Highway, Crawley, Western Australia,6009, Australia. e-mail: jingbo,rachel@csse.uwa.edu.au

Please use the following format when citing this chapter:

Sun, J., Cardell-Oliver, R., 2008, in IFIP International Federation for Information Processing, Volume 265, Advances in Ad Hoc Networking, eds. Cuenca, P., Guerrero C., Puigjaner, R,, Serra, B., (Boston: Springer), pp. 37-48. 
is to develop autonomic sensor networks. Although researchers have proposed many protocols to improve the data delivery reliability in wireless sensor networks $[2,3$, $8,18,21]$, no one alone can really adapt to changeable environmental conditions. For autonomy, different data delivery strategies are required for different radio link conditions.

The technologies used to guarantee reliable data delivery using single path usually are retransmissions, which include retransmissions based on positive acknowledgements and retransmissions based on negative acknowledgements. Multiple paths delivery is another mechanism used for providing reliable data delivery over wireless links.

In this paper, we choose three protocols: timeout-based protocol [10], streaming protocol [3] and ExOR protocol [2], which represent the above three technologies respectively. In choosing optimal protocol, we need to consider memory cost (buffer size), latency to successfully deliver a packet and energy expended per packet delivered. For space, we only analyse the energy consumption of these three protocols in different environments in this paper. Our results help to identify situations in which we should use each of these protocols to optimise reliability in wireless sensor networks. We find streaming performs better than ExOR when forward link quality is good (above 0.5 ); while ExOR performs better when forward link quality is from medium to low (below 0.5).

Based on analysis results, we propose an autonomic communication framework (ACF) for sensor networks that can change its behaviour according to different environments. ACF includes protocol library, monitor module, scheduler module and central control service. The central control service decides the protocol used in the running sensor network based on the gathered information from the monitor module, and controls the node behaviour by using scheduler module. Thus, the network can use different mechanisms under different situations so that the network performance can be improved. Experimental results show that our framework performs better than ExOR in terms of link efficiency and energy consumption.

We focus on sensor networks that require reliable transmission of batches of data, for example data gathered by periodic monitoring, for which delivery delays can be tolerated. In this paper we focus on single hop communication, for example between nodes and cluster heads. Many sensor network applications, such as environmental monitoring, satisfy these criteria.

The reminder of this paper is organized as follows. Section 2 analyses the performance of the timeout-based protocol, streaming protocol and ExOR protocol in different environmental conditions. In Section 3 we propose an autonomic communication framework (ACF) for sensor networks based on the analysis from Section 2. The experiment setup and results are presented in Section 4. Section 5 describes related work. Section 6 concludes this paper and outlines our future work. 


\section{Protocol Performance Analysis}

In this section, we briefly introduce three reliable protocols: timeout-based protocol, streaming protocol and ExOR protocol, then point out limitations of these three protocols in terms of energy consumption.

\subsection{Protocol Overview}

In the timeout-based protocol [10], the source node relies on a timer to control retransmissions. The source sends one packet at a time and sets a timer. If the source receives an acknowledgement from the destination, the next packet can be transmitted. Otherwise, the source retransmits the packet one or more times when the timer expires, up to some limit or an acknowledgement is received, before the packet is declared lost.

In streaming [3], the source node sends packets in sequence. The destination replies with a Retransmission Request Packet (RRP) if it finds a sequence gap in the received packets. Streaming is more efficient than the timeout-based protocol since the destination only sends a negative acknowledgement (NACK) for every lost packet, instead of sending a positive acknowledgement for every received packet, which reduces the amount of transmissions. However, streaming still needs to wait a NACK for every transmitted packets. Thus, using aggregated NACKs or batch transmissions can improve the efficiency of streaming. The buffer size of streaming is unpredictable. When the link quality is getting worse, its buffer size should be increased in order to store all non-sequence packets.

In ExOR [2], source sends a batch of packets to destination via a named multihop path and those packets may be overheard by potential forwarder nodes. The destination replies with multiple copies of its acknowledgement. The forwarder then forwards packets which are received by itself but not by the destination. After that, the source retransmits remaining packets. This procedure is repeated until all packets are received by the destination. ExOR is a multihop protocol in which source has global information about routes. In this paper we use ExOR as single link. ExOR is more suited to bad link quality environments than streaming because it uses multiple paths to improve reception. However, when link quality is good, it consumes more energy because of its redundant paths.

\subsection{Energy Consumption Analysis}

In this section, we compare the performance of the timeout-based protocol, streaming protocol and ExOR protocol in terms of energy consumption. Formally, we define link quality between two nodes in terms of the probability of successful packet delivery from one node to the other. For sensor nodes $\mathrm{A}$ and $\mathrm{B}$, link quality, $L Q_{A B}$, 


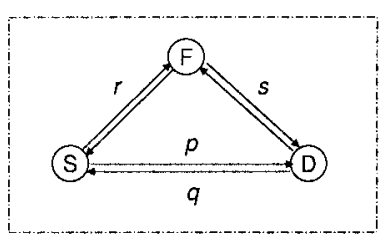

Fig. 1 Timeout, Streaming and ExOR Protocol Network Architecture

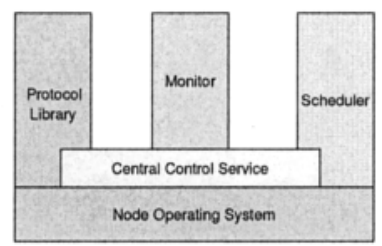

Fig. 2 Autonomic Communication Framework Architecture

at a given time is denoted by a pair $(p, q)$ giving the probability of successful packet delivery from $A$ to $B$ and $B$ to $A$ respectively. Figure 1 shows nodes and links used for timeout (SD), streaming (SD) and ExOR (SDF).

Energy Consumption is an important criteria to be considered in the design of sensor network protocols. It can be measured by average energy consumption per delivered packet ( $A E C$ for simplicity in the following) that depends on the time used for successfully delivering one packet from source to destination.

Suppose each node uses the same energy. The following parameters are used for calculating $A E C$.

- $T x D_{i}$ : the time used for node $i$ to transmit or listen for a batch of data packets;

- $T x A_{i}$ : the time used for node $i$ to transmit or listen for acknowledgement(s);

- $C N$ : the electrical current of a node when its radio is on;

- $V N$ : the battery voltage of a node;

- $N$ : the number of nodes participating in the protocol;

- $B S$ : the number of data packets transmitted in one cycle.

For each node, energy consumption is the energy (that is volts $\times$ amperes) times the time of its radio turned on in order to deliver or listen for a packet. The total energy consumption is the sum of the energy used by each participating node. Therefore, the $A E C$ is the total energy consumption of all participating nodes divided by the number of data packets transmitted during this period:

$$
A E C=N \times\left(C N \times V N \times\left(T x D_{i}+T x A_{i}\right)\right) / B S
$$

In the timeout protocol, both source and destination have the same radio on time. Suppose the batch size is one. The packet delivery success ratio for the source is $p q$ because success means both data packet and acknowledgment are successfully received. Therefore, the source sends $1 / p q$ times on average to know one packet has been successfully received. The average duration per packet is $\left(T x D_{S}+T x A_{D}\right) / p q$. The $A E C$ of the timeout protocol is therefore:

$$
A E C_{\text {timeout }}=2 \times C N \times V N \times \frac{1}{p q}\left(T x D_{S}+T x A_{D}\right)
$$

Using the same assumption as the timeout protocol, in streaming, the possibility of packet loss is $1-p$ and the average number of RRPs sent by the destination node 
is $1 / p q$. The average number of retransmissions for a lost packet sent by the source is $(1-p) \frac{1}{p q} \times q=\frac{1}{p}-1$. The total transmission times (including retransmissions) is $1 / p$. Therefore, the $A E C$ is:

$$
A E C_{\text {streaming }}=2 \times C N \times V N \times \frac{1}{p}\left(T x D_{S}+T x A_{D}\right)
$$

The $A E C$ of streaming is not related to backward link quality because lost packets are detected as long as the network runs long enough and the buffer is large enough to store all out of order packets. However, its energy is still wasted by listening for a NACK even when none is transmitted. We also note that the cost of streaming's energy use is high buffer requirement and high packet delivery latency when the link quality is low. The energy efficiency of streaming could be improved by sending data packets in batches. Because of the limited space, we do not give a detailed analysis in this paper.

In ExOR, the $A E C$ depends on how many total transmissions are used by the source and forwarder to successfully transmit a batch of packets to the destination. Here, we do not consider the backward link quality because the destination sends multiple copies of acknowledgments (10 in [2]) in order to make sure source or forwarder is able to receive at least one. Three nodes, source, forwarder and destination, are used in this single-hop network with radios turned on at all times. The average number of transmission times, including retransmissions is $\frac{1}{p+r s-p r s}$. Suppose the data transmission time of the forwarder is the same as that of the source, the estimated $A E C$ of the ExOR protocol is:

$$
A E C_{E x O R}=3 \times C N \times V N \times \frac{1}{p+r s-p r s}\left(T x D_{S}+T x D_{F}+T x A_{D}\right) / B S
$$

The electrical current of Fleck nodes [17], which are used in our implementation, is measured in terms of different states. Table 1 from [1] shows that node uses most of its energy when its radio is on. In its low power mode, the energy consumption is negligible, which supports our $A E C$ estimation.

Table 1 Power Usage Table for Fleck Nodes [1]

\begin{tabular}{cc}
\hline Mode & Current $(\mathrm{mA})$ \\
\hline Low Power & 0.4 \\
Sensing Only & 14.0 \\
Radio Only & 35.8 \\
\hline
\end{tabular}

In order to get a clear view of the energy consumption for three protocols, we graph Equation (2), (3) and (4) based on parameters in Table 2. Since ExOR sends 10 copies of the acknowledgement, the TxAD for ExOR is 10 times as that for the timeout and streaming protocol. 


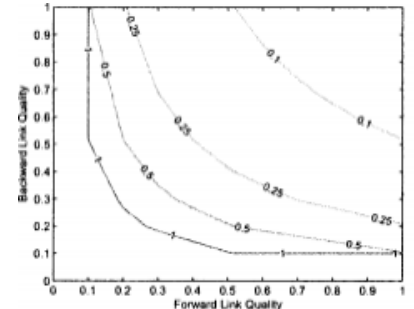

(a)

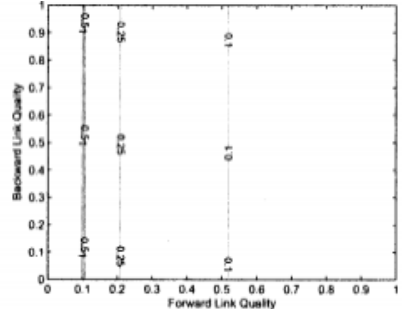

(b)

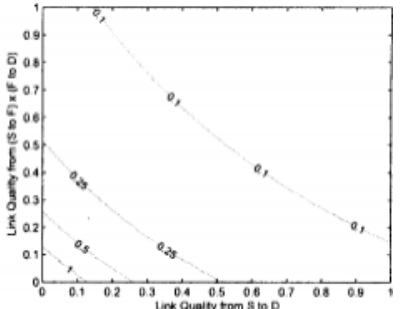

(c)

Fig. 3 Distribution of $A E C$ (Joule): (a) Timeout; (b) Streaming; (c) ExOR.

Table 2 Parameters Used for Calculating $A E C$

\begin{tabular}{|c|c|c|c|c|c|}
\hline & $\mathrm{TxD}(\mathrm{ms})$ & $\mathrm{xA}(\mathrm{ms})$ & $\mathrm{CN}(\mathrm{mA})$ & $\mathrm{N}(\mathrm{V}$ & N BS \\
\hline Timeout & 200 & 100 & 35.8 & 2.4 & 21 \\
\hline Streaming & 200 & 100 & 35.8 & 2.4 & 2 \\
\hline ExOR & 2000 & 1000 & 35.8 & 2.4 & 310 \\
\hline
\end{tabular}

Figure 3 shows the distribution of energy consumption of three protocols measured in units of Joule. Comparing Figure 3(b) with Figure 3(c), we observe that the energy consumption of streaming is better than that of the ExOR protocol when its forward link quality is above 0.5. However, ExOR is better when forward link quality is below 0.5 even though it uses three nodes instead of two. The forwarder is indeed able to help increase the delivery speed without consuming much energy. The energy consumption of the timeout protocol is worst according to Figure 3(a), 3(b) and 3(c), because it needs good quality of both forward and backward links in order to reduce the number of retransmissions.

\section{Autonomic Communication Framework for Sensor Networks}

Based on the above analysis, we observe that in order to adapt to varying environments, different mechanisms should be used under different situations. Only an autonomic network, which can alternate its behaviour, can really perform well in time varying environments. Therefore, we propose an autonomic communication framework (ACF), where nodes can dynamically change their behaviour according to current environmental conditions.

The architecture of ACF contains four modules: protocol library, monitor, scheduler and central control service, shown in Figure 2. The protocol library has protocols which may be needed in different situations. The monitor module is used to monitor the change of the situation, which will result in the change of the protocol. 
Node behaviour controlled by the scheduler module, includes sending, receiving, waking up and sleeping. All the information is passed to nodes by the central control service module. We will specifically describe the function of these modules in the following.

\section{Protocol Library}

The protocol library contains implementations of protocols to be used under different environmental conditions. The protocol switching time is decided by the monitor module based on the protocol switching threshold. That threshold is defined by users before the start of the experiment. Our library implements versions of the streaming and ExOR protocols. When nodes use these protocols, they transmit batches of packets since this strategy increases transmission speed and reduces waiting time. Acknowledgements are aggregated in one packet for every batch. The number of the acknowledgements for both protocols is changed dynamically based on the current link quality.

\section{Monitor Module}

The monitor module is used to monitor current conditions, such as battery level, sensor temperature, link quality and solar power. In our experiments, we use link quality as the main metric to decide if the node's behaviour should be changed. We can use any existing link quality estimation method, such as ETX [7] or window mean with EWMA [22]. In our implementation, we use a simple estimation method in order to reduce the complexity of the implementation and avoid using much memory and calculation energy. Each node estimates link quality by counting packets received from neighbours during a fixed time window.

\section{Scheduler Module}

This high-level scheduler is used to decide upon the node behaviour, such as transmitting, receiving, listening, waking up and sleeping for every node in the network. It can be used to schedule communication over multiple links. Every node has its own time slot to transmit and receive packets. We have implemented a simple fixed schedule. The schedule frame of each node is built as follows. Each node is assigned a transmitting slot by the base station and listens to the traffic from its neighbours during other slots. Then the node marks the specific slot as listening for one of its neighbours when it hears traffic from that neighbour during that slot. Transmitting and listening slots have the same length. In our implementation, each node has a 5-second time slot in the schedule frame to transmit a batch of packets, and uses the other two slots for listening or sleeping.

Synchronization is also controlled by the scheduler module. We integrate a lightweight synchronization scheme in which data packets carry a time stamp and children update their times to match their parents [14].

\section{Central Control Service}

The central control service receives information from the monitor module and decides which protocol should be chosen from the protocol library based on the 
predefined protocol switching threshold. A threshold indicator is integrated in the data packets. The scheduler module uses the threshold indicator to control node behaviour. In our implementation, based on the previous analysis, when the link quality is below 0.5, the ExOR protocol is chosen, otherwise streaming is selected. When using ExOR, the central control service also passes information to scheduler that one more node should be woken up during the source transmission slot.

\section{Results}

\subsection{Experiment Set $U p$}

We implemented our autonomic communication framework ACF and ExOR using TinyOS/nesC [15] with TinyOS Blocking Library [13]. We evaluated their performance in a single hop network using CSIRO Fleck motes [17].

Our experiments used a source, a destination and a helper node, with a fourth node with high gain antenna as the base station. The base station was connected to a personal computer and the three nodes were placed in two different rooms, with the destination in a separate room from the source and the helper. We put nodes in different angles in order to get different link qualities.

The schedule slot was 5 seconds and the size of the schedule frame was 3 . The monitor module performed every 30 minutes to calculate link quality between the source and the destination. The batch size was 10 . The protocol switching threshold was 0.5 based on bidirectional link quality according to the above three protocol analysis. The destination node sent 2 copies of acknowledgements when link quality was above the threshold, otherwise it sent 6 copies. The base station recorded all packets it heard from the three nodes.

In our experiments, the length of the data packet was the same as the length of the acknowledgement packet ( 33 bytes), although in practice the length of the acknowledgement could be much shorter. The smaller acknowledgements only affect the best performance of the network, but do not affect the trend of the performance over varying link quality.

We repeated each experiment at least 3 times in different days in order to enlarge the range of link quality. Two experiments ran total 46.5 hours together. In order to get fair comparison, data chosen in Table 3 was the average from data that had the first two same decimal digits. The forwarder was in the middle of the source and the destination and so its link quality to these two nodes were good enough to fit for our purpose. The experimental results are with $95 \%$ confidence interval. 
Table 3 Link Quality: Autonomic Communication Framework (ACF) and ExOR

\begin{tabular}{cccccccccccccc}
\hline Link Protocol & L1 & L2 & L3 & L4 & L5 & L6 & L7 & L8 & L9 & L10 & L11 & L12 \\
\hline SD & ACF & 0.241 & 0.314 & 0.375 & 0.408 & 0.505 & 0.666 & 0.752 & 0.762 & 0.778 & 0.803 & 0.823 & 0.892 \\
& ExOR & 0.242 & 0.312 & 0.378 & 0.416 & 0.507 & 0.669 & 0.755 & 0.761 & 0.779 & 0.808 & 0.826 & 0.890 \\
\hline \multirow{2}{*}{ DS } & ACF & 0.659 & 0.758 & 0.747 & 0.751 & 0.785 & 0.867 & 0.895 & 0.702 & 0.878 & 0.899 & 0.983 & 0.952 \\
& ExOR & 0.350 & 0.769 & 0.859 & 0.575 & 0.933 & 0.960 & 0.796 & 0.998 & 0.801 & 0.912 & 0.913 & 0.828
\end{tabular}

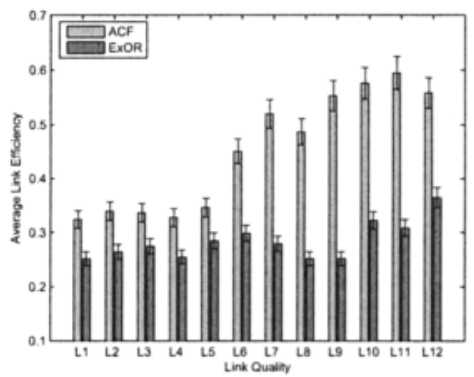

Fig. 4 Link Efficiency: ACF and ExOR

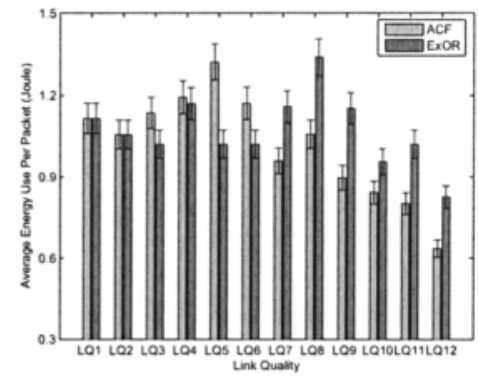

Fig. 5 Energy Consumption: $\mathrm{ACF}$ and ExOR

\subsection{Experimental Results}

In this section, we analyse the performance of our framework ACF from two aspects: link efficiency and energy consumption.

The $x$-axis of Figure 4 and 5 corresponds to categories in Table 3. From Figure 4 we observe that the link efficiency of ACF is better than that of ExOR, especially when link quality is above 0.5. It improves the link efficiency than ExOR up to $119 \%$. The main reason is that it changed its behaviour by using 2 nodes instead of 3 nodes in the ExOR protocol when the bidirectional link quality between the source and the destination is above the predefined threshold. Another reason is that the number of acknowledgements used in our framework is dynamically changed to adapt to its environment, which decreases the total number of packets transmitted in the network.

We also compared the energy consumption of ACF with ExOR shown in Figure 5. It shows that $\mathrm{ACF}$ performs better than ExOR when link quality is above the threshold and has almost the same performance as the ExOR when link quality is below the threshold. We also notice that the energy consumption of our framework is worse than that of ExOR when link quality is around the threshold in Figure 5. That may be because of the bias of the estimated link quality or because the current threshold may not be optimal. However, the overall performance of our framework is still better than that of ExOR. 
Based on the above analysis, our autonomic communication framework ACF has the optimal overall performance based on the analysis of its link efficiency and energy consumption.

\section{Related Work}

Reliable delivery is one of important issues in wireless sensor networks explored by researchers. Retransmission is a major mechanism used to provide reliable delivery in sensor networks. PSFQ [21] and RMST [18] employ a hop-by-hop negative acknowledgement (NACK) mechanism either at the link or transport layer to increase network reliability. In PSFQ, the user broadcasts data segments with sequence numbers to its neighbors periodically. The receiver relays these segments to its neighbours if there is no sequence gap in received segments. Otherwise, it requests immediate retransmission of the missing segments using a NACK message. RMST improves on PSFQ by using MAC layer retransmission instead of only relying on explicit NACKs from the transport layer to recover lost packets. However, both of them route data along a single path and so are vulnerable to low link quality anywhere in that path.

Multiple path delivery protocols improve on the reliability of single path protocols. ReInForM [8] provids reliable data delivery to adapt to the channel error rate by using multiple copies of the same packet delivered through multiple edgedisjoint paths from source to sink. Nodes themselves decide their forwarding directions without explicit paths. However, the ReInForm's requirement of the existence of multiple edge-disjoint paths in connected unit disk graphs of uniform density is unrealistic in the real world. The main idea of GRAB [23] is that a packet is forwarded through interleaved multiple paths from source to sink. The number of paths is determined by the required reliability, which is used to control the degree of path redundancy. Because of multiple paths are created on the fly, all nodes in GRAB have to listen to packets from their neighbours, this increases the overall energy use.

Several self-healing approaches exist for ad hoc and sensor networks, such as CATS [16] and the autonomic routing framework (ARF)[12]. CATS is a cross-layer approach to help routing protocol maintain network reliability. It uses a Management Plane to gather information in order to identify and react to network failure. However, the amount of information from each of the seven layers of the OSI Reference Model generates too much traffic for a sensor network setting. The ARF aims to improve the adaptivity of routing services in sensor networks by adapting to the application and network dynamics. The aim of ARF is different from ours. ARF maintains some feature functions that are decoupled from the routing service. Feature functions have a set of parameters to tune to fit application and network changes. ARF uses its monitor module to collect state information and make adaptation decision. However, ARF is implemented on a Linux PC and its total code size is around $180 \mathrm{~KB}$, and so it is not suited to memory constrained sensor nodes. Our Fleck nodes only have $128 \mathrm{~KB}$ programmable flash memory. 
ASCENT [5] is a topology-adaptive protocol, which uses redundancy to extend network lifetime. A minimum set of nodes is selected to establish a routing backbone. In ASCENT, each node assesses its connectivity and adapts its participation in the multihop network topology based on the measured operating region. ASCENT is neither a routing nor data dissemination protocol. It only decides upon the network topology. Gu et al. proposed a dynamic switch-based forwarding protocol (DSF) [11] that reduces end-to-end latency over unreliable links. DSF uses multiple potential forwarding nodes at each hop that in turn receive packets from the source. This strategy overcomes unreliable links and reduces transmission delay. Although DSF also uses extra nodes for forwarding, its aim minimising latency is different from our aim minimising energy use.

\section{Conclusion}

Reliable energy efficient data delivery is an important function of wireless sensor networks, particulary in harsh environments. Our goal is to build a reliable, adaptive data delivery approach which maximises the field life of environmental monitoring sensor networks.

We analysed the performance of existing reliable protocols: timeout-based protocol, streaming protocol and ExOR protocol in order to find out if current technologies used for improving the reliable data delivery alone can really adapt to varying environments. Analysis of energy consumption shows that none of these protocols is able to optimise its performance by adapting completely to varying environments.

In this paper, we present an autonomic communication framework (ACF) for sensor networks that is able to optimise its performance for time-varying links. ACF uses different mechanisms based on the different environmental conditions in order to achieve an optimal performance. We implemented this framework and ExOR on CSIRO Flecks to test their real world performance. Experimental results show that the overall performance of our framework is better than that of ExOR. In the future, this framework will be extended for multi-tier networks and tested in the field at different sensor network scales.

\section{References}

1. Wireless sensor networks project wiki. URL http://www.csse.uwa.edu.au/ $\sim \mathrm{mkranz} /$ wiki/index.php/Fleck:Fleck_Power

2. Biswas, S., Morris, R.: ExOR: opportunistic multi-hop routing for wireless networks. In: SIGCOMM '05: Proceedings of the 2005 conference on Applications, technologies, architectures, and protocols for computer communications, pp. 133-144 (2005)

3. Cao, Q., He, T., Fang, L., Abdelzaher, T., Stankovic, J., Son, S.: Efficiency centric communication model for wireless sensor networks. In: IEEE INFOCOM (2006) 
4. Cardell-Oliver, R., Smettem, K., Kranz, M., Mayer, K.: A reactive soil moisture sensor network: Design and field evaluation. International Journal of Distributed Sensor Networks pp. 149-162(2005)

5. Cerpa, A., Estrin, D.: ASCENT: Adaptive self-configuring sensor networks topologies. IEEE Transaction on Mobile Computing 3(3), 272-285 (2004)

6. Cerpa, A., Wong, J., Potkonjak, M., Estrin, D.: Temporal properties of low power wireless links: Modeling and implications on multi-hop routing. In: Proceedings of the 6th ACM international symposium on Mobile ad hoc networking and computing, pp. 414 425 (2005)

7. Couto, D.S.J.D., Aguayo, D., Bicket, J., Morris, R.: A high-throughput path metric for multihop wireless routing. In: MobiCom '03: Proceedings of the 9th annual international conference on Mobile computing and networking, pp. 134-146 (2003)

8. Deb, B., Bhatnagar, S., Nath, B.: RelnForM: Reliable information forwarding using multiple paths in sensor networks. In: $\mathrm{LCN}$ '03: Proceedings of the 28th Annual IEEE International Conference on Local Computer Networks, p. 406 (2003)

9. Falchi, A.: Sensor networks: Performance measurements with motes technology. Master's thesis, Dept. of Information Engineering, Uni. of Pisa (2004)

10. Gnawali, O., Yarvis, M., Heidemann, J., Govindan, R.: Interaction of retransmission, blacklisting, and routing metrics for reliability in sensor network routing. In: Proceedings of the First IEEE Conference on Sensor and Adhoc Communication and Networks (2004)

11. Gu, Y., He, T.: Data forwarding in extremely low duty-cycle sensor networks with unreliable communication links. In: SenSys '07: Proceedings of the 5th international conference on Embedded networked sensor systems (2007)

12. He, Y., Raghavendra, C.S., Berson, S., Braden, R.: An autonomic routing framework for sensor networks. In: Cluster Computing, Specical Issue on Autonomic Computing (2006)

13. Kranz, M.: Tinyos blocking library. URL http://wsnwiki.csse.uwa.edu au/index.php/Software

14. Lee, W.L.: Flexible-schedule-based tdma protocols for supporting fault-tolerance, on-demand tdma slot transfer, and peer-to-peer communication in wireless sensor networks. Ph.D. thesis, The University of Western Australia (2007)

15. Levis, P., Madden, S., Polastre, J., Szewczyk, R., Whitehouse, K., Woo, A., Gay, D., Hill, J., Welsh, M., Brewer, E., Culler, D.: Ambient Intelligence, chap. TinyOS: An Operating System for Sensor Networks, pp. 115-148. Springer Berlin Heidelberg (2005)

16. Sadler, C.M., Kant, L., Chen, W.: Cross-layer self-healing mechanisms in wireless networks. In: Proceedings of the World Wireless Congress (2005)

17. Sikka, P., Corke, P., Overs, L.: Wireless sensor devices for animal tracking and control. In: Proceedings of the 29th Annual IEEE International Conference on Local Computer Networks, pp. $446-454$ (2004)

18. Stann, F., Heidemann, J.: RMST: Reliable data transport in sensor networks. In: Proceedings of the First International Workshop on Sensor Net Protocols and Applications, IEEE (2003)

19. Sun, J., Cardell-Oliver, R.: An experimental evaluation of temporal characteristics of communication links in outdoor sensor networks. In: ACM REALWSN'06, pp. 73-77. Uppsala, Sweden (2006)

20. Szewczyk, R., Mainwaring, A., Polastre, J., Anderson, J., Culler, D.: An analysis of a large scale habitat monitoring application. In: Proceedings of the 2 nd International Conference on Embedded Networked Sensor Systems, pp. 214-226 (2004)

21. Wan, C.Y., Campbell, A.T., Krishnamurthy, L.: PSFQ: a reliable transport protocol for wireless sensor networks. In: WSNA '02: Proceedings of the 1st ACM international workshop on Wireless sensor networks and applications, pp. 1-11 (2002)

22. Woo, A., Tong, T., Culler, D.: Taming the underlying challenges of reliable multihop routing in sensor networks. In: Proceedings of the Ist international conference on Embedded networked sensor systems, pp. 14-27 (2003)

23. Ye, F, Zhong, G., Lu, S., Zhang, L.: GRAdient broadcast: a robust data delivery protocol for large scale sensor networks. Wirel. Netw. 11(3), 285-298 (2005)

24. Zhao, J., Govindan, R.: Understanding packet delivery performance in dense wireless sensor networks. In: Proceedings of the 1st international conference on Embedded networked sensor systems, pp. 1-13 (2003) 